\title{
Study on Technical, Behavioral and Organizational Factors Affecting the Performance of Mother and Child Tracking System in Rural Area of Varanasi District, Uttar Pradesh: A Qualitative Study
}

Pooja Verma ${ }^{1}$, Sangeeta Kansal ${ }^{2}$, Madhutandra Sarkar ${ }^{3}$, Nishu Kesh ${ }^{4}$, Alok Kumar ${ }^{5}$

${ }^{1}$ PhD Scholar, Banaras Hindu University, Varanasi, Uttar Pradesh, India; ${ }^{2}$ Professor and Head, Department of Community Medicine, Institute of Medical Sciences, Banaras Hindu University; ${ }^{3}$ Medical Officer, Department of Community Medicine, Institute of Medical Sciences, Banaras Hindu University; ${ }^{4} \mathrm{PhD}$ Scholar, Banaras Hindu University, Varanasi, Uttar Pradesh, India; ${ }^{5}$ Associate Professor, Department of Statistics, Banaras Hindu University, Varanasi, Uttar Pradesh, India.

\begin{tabular}{|c|c|c|c|c|c|c|c|c|}
\hline Abstract & Introduction & Methodology & Results & Conclusion & References & Citation & \multicolumn{2}{|c|}{ Tables / Figures } \\
\hline \multicolumn{9}{|c|}{ Corresponding Author } \\
\hline \multicolumn{8}{|c|}{$\begin{array}{l}\text { Dr. Sangeeta Kansal, Department of Community Medicine, Institute of Medical Sciences, Banaras Hindu } \\
\text { University, Varanasi-221005, Uttar Pradesh, India. } \\
\text { E-mail ID: sangeetakansalbhu@gmail.com }\end{array}$} & 回部四 \\
\hline
\end{tabular}

\section{Citation}

Verma P, Kansal S, Sarkar M, Kesh N, Kumar A. Study on Technical, Behavioral and Organizational Factors Affecting the Performance of Mother and Child Tracking System in Rural Area of Varanasi District, Uttar Pradesh: A Qualitative Study. Indian J Comm Health. 2020;32(2):423-426.

Source of Funding: Nil Conflict of Interest: None declared

\section{Article Cycle}

Received: 31/03/2020; Revision: 15/04/2020; Accepted: 05/05/2020; Published:30/06/2020

This work is licensed under a Creative Commons Attribution 4.0 International License.

\section{Abstract}

Background: The Mother and Child Tracking System (MCTS) portal captures and tracks all pregnant women right from conception up to 42 days after delivery and all children below five years. Objectives: This study was conducted to find out the technical, behavioral and organizational factors that affect the process and performance of MCTS. Material and Methods: This was an exploratory qualitative study conducted in Varanasi district by using multistage random sampling method. Data were collected by in-depth interviews of 48 health care providers. Results: Majority of the ANMs and ASHAs were not aware of the meaning of "Tracking System" and the use of MCTS ID. Inappropriate training of ASHAs, absence of supervision and monitoring by ANMs, overburdened DEOs and ANMs, poor internet connection, slow server speed, software problem and frequent power failures were identified as major factors for poor performance of MCTS. Conclusion: The most effective measure to improve the performance of MCTS would be to properly train grass root level workers so that more efficient data can be fed into MCTS.

\section{Keywords}

MCTS; Health Care Providers; Training; Performance; Qualitative

\section{Introduction}

The Mother and Child Tracking System (MCTS) is a webbased portal launched by the Government of India in December 2009 in collaboration with the States and the Union Territories. Name-based data of mothers and children are uploaded online through MCTS portal. This ambitious project captures and tracks all pregnant women right from conception up to 42 days after delivery and all children up to five years of age.(1) It has been declared as a Mission Mode Project under the National e-Governance Plan (NeGP) in July 2011.(2) The data on pregnant women and children are captured by the local accredited social health activist (ASHA) and auxiliary nurse cum midwife (ANM) of the area on a tracking format which include, apart from other information, contact number of the mother to reach her through short message service (SMS).

All the information are uploaded on MCTS portal by the data entry operator (DEO). The data are compiled at the district level and propagated to the national level.(3)

Previous studies $(4,5,6)$ identified inappropriate training, overburdened DEO, poor internet connection, slow server speed, poor electricity supply, non-standardized registers and formats as the factors affecting the performance of MCTS. There is dearth of correct data on the current status of MCTS available from the eastern part of Uttar Pradesh. 


\section{Aims \& Objectives}

To find out the technical, organizational and behavioral factors affecting the process and performance of MCTS in the rural area of Varanasi district.

\section{Material \& Methods}

Study type and Study duration: This exploratory qualitative study was conducted between January to December 2017 in three blocks of Varanasi district.

Sampling procedure: Out of 8 blocks of Varanasi district, 3 blocks were randomly selected. Then, one PHC was selected randomly from each block. Further, two subcenters (SCs) were randomly selected from each PHC.

Data collection: In-depth interviews of the health care providers (HCPs) were conducted to know about their views on the factors affecting the process and performance of MCTS and the problems associated with it. In total, $48 \mathrm{HCPs}$ were interviewed, including 36 ASHAs and 6 ANMs at the SC level and 3 medical officers-incharge (MOICs) and 3 DEOs at the PHC level. A semistructured questionnaire guide was used which was developed to serve the objectives of the study. The question guide included the prompts related to interviewee's knowledge about the tracking system, the utility of MCTS portal for tracking mother and child and problems faced by them in using the portal. Before the interview, the purpose and the objectives of the study were explained to each interviewee and consent was taken. The interviews were conducted at the workplace of the respective HCPs and lasted for about 45 minutes to 1 hour. With the permission of interviewees, audiorecording of the interviews was done on mobile phones and field notes were taken. All the interviews were conducted in Hindi.

Data analysis: Data were transcribed in verbatim. These units and responses generated themes and subthemes and improved the question guide further until data saturation occurred. The transcripts were read and a coding framework was prepared, and finally analysis was done.

Ethical approval: Ethical approval for the study was obtained from the Institute Ethics Committee.

\section{Results}

Based on the interviews with HCPs and the experience of the researchers, the results are presented as below:

Knowledge about tracking system: Majority of the ANMs and ASHAs were not aware about the meaning of "Tracking System", although they were well aware with the services covered under MCTS.

One ASHA quoted, "Tracking kamatlabjachaaa or bachaako sari suvidhadenahotahai."

Knowledge about MCTS ID and its utility: Majority of the ANMs were not aware about the use of MCTS ID. Only one ANM said that the ID was important for delivery and the other said that the pregnant female could easily access any service from any government health facility with this ID.

One another ANM quoted, "ID kebina Janani Suraksha Yojanaka paisa nahimiltahai."

Majority of the ANMs and ASHAs were not writing ID in the mother and child protection (MCP) card. Although they were recorded in their own registers. One of the ASHA informed that majority of the ANMs were reluctant to provide ID to them.

Message alerts/calls: Majority of the ANMs told that they got MCTS ID only from the messages. Most of the ASHAs said that they received recorded calls of Dr. Anita for the knowledge about mother and child health irregularly. The irregularity of the calls was a very big obstacle to provide correct knowledge to HCPs about MCTS.

Complexity of format/register: According to most of the ASHAs and ANMs, the given reproductive and child health $(\mathrm{RCH})$ register was having many new columns which were not present in the earlier register. ASHAs were having difficulty in filling the registers because they had not received any formal training to fill the register.

This is supported by quote of one ASHA, "Registeritnabada de diyagayahai, kahi le keaanejaane me dikkathotihai, register bharne me bhidikkataatihai."

Problem related to software: As MCTS portal was slightly different from $\mathrm{RCH}$ register, DEOs were unable to upload the data properly in the portal. To overcome these difficulties, MCTS portal was being converted into $\mathrm{RCH}$ portal.

One DEO quoted, "Pehle MCTS tracking portal tha, ab usko $\mathrm{RCH}$ me badaldiyagayahai, jiskewajha se data upload karne me dikkat aa rahihai, kabhikabhi to portal support hi nahikartahai."

Inadequate training on tracking system: The finding of the study shows that the main reason for incompleteness of data and its bad quality was lack of appropriate training of HCPs.

a. All DEOs had received only one to two days of training in filling the data in MCTS portal.

b. Majority of the ANMs had received one to two days of training in every second and fourth month to fill the data in the register.

c. No one of the ASHAs had received any training to fill the tracking registers. ASHAs informed that they were filling their registers with the help of ANMs.

One ANM quoted,"ASHA-o koaapnesamnebaithake register bharwatehai, bar barbatane par bhigalatbhardetihai."

No dedicated staff/DEO: One DEO was posted at each block PHC and community health center (CHC). However, there was no dedicated DEO for MCTS data entry.

Availability of resources: Internet connectivity and power supply were irregular at all the PHCs. Many DEOs cumulatively logged into the portal at the working hours, which considerably slowed down the server and delayed timely data entry. An online data upload portal should be 
INDIAN JOURNAL OF COMMUNITY HEALTH / VOL 32 / ISSUE NO 02 / APR - JUN 2020 supported by a strong server to decrease delays in data entry.

One DEO quoted, "Computer par entry walajitnabhikaamhai, saarakaam DEO kemattheehotahai, upor se kabhi light chalijatihai to kabhi network kharab ho jatahai."

Duplication of data: According to DEOs, portal was not connected with other blocks and districts. Most of them said that if the pregnant woman moved to the mother's house, then a new ID was generated to her at the concerned block which caused duplication of data.

This is supported by quote of one DEO, "Pregnantaurate delivery ke time apnemaykechalijatihai. Bina ID ke block par delivery nahihotihai, isliyeunkowahabhiek ID de di jatihai, jisseek hi auratki 2 ID ho jatihai. Ab wo waha delivery karaletihai, to dusre block par uski service due dikhatahai."

No monitoring/feedback: All the ASHAs had not received any kind of feedback regarding the mistakes made in the register and as a result they could not improve their mistakes. Underutilization of data and poor feedback from the higher officials had been recognized as major gaps.

One DEO quoted, "Meeting me register kigaltiyoke bare me batanachahiye."

Difficulties in obtaining MCTS ID and due list: Majority of the ASHAs and ANMs informed that they were having difficulties in getting MCTS ID. As per the guidelines, they submitted their registers or tracking format to DEOs to enter the data in the portal. DEOs did not provide MCTS ID immediately. According to them, they had to go to the block PHC several times to take MCTS ID, which affected their field work and put financial burden on them.

One ANM said, "Hume ID lenekeliye kai baar block par jaanapadtahai. Jiskewajha se humarabahutsaarakaamnahi ho patahai."

MCTS has an inbuilt mechanism for generating a due list of pregnant females and children before each immunization session. However, most of the ASHAs and ANMs were complaining that the due list was being prepared by them. They received only a blank format of due services from the block in which they wrote the due services of pregnant females and children after each Village Health and Nutrition Day (VHND) session.

Completeness of data: All DEOs informed that they received incomplete registers from ASHAs/ANMs. Moreover, they were getting registers weekly to fill the data, which had increased their workload.

No motivation of staff: There are many factors that hinder health workers to work properly.

a. Not competent to fill $\mathrm{RCH}$ register: Filling $\mathrm{RCH}$ register is the job of ANM, but in practice, ASHA fills $\mathrm{RCH}$ register.

b. Financial burden: According to majority of the ASHAs and ANMs, DEOs returned the registers to them in case information were incomplete or there was any
[Study on Technical...] | Verma P et al confusion. This put financial burden to them because of their frequent travel to PHC.

This is supported by quote of one ASHA, "ANM didi to baarbaar ASHA logo ko hi register lenebhejdetihai ,aanejaane me paisa bahutkharchhotahai. Humara fieldkakaam me bhidikkataatihai."

c. Workload of maintaining record of formats and registers: Majority of the ASHAs and ANMs preferred to maintain a personal register to fill information and later on transferred it to the main $\mathrm{RCH}$ register. They spent daily two to three hours to maintain the registers, which put extra work burden on them.

Difficulties faced by health workers in getting mobile numbers of beneficiaries: ASHAs and ANMs informed that pregnant women didn't have personal mobile phones, and they mostly gave the number of their husbands or other family members. Some of them were not willing to share their numbers also. This caused delay in uploading their contact numbers in the portal. ASHAs were writing their own mobile numbers in the register.

One ASHA quoted, "In logan se number maagatmaagatthakjaawathai, par in logon kekaan par junaregat baa."

\section{Discussion}

The present study showed that majority of the ANMs was not aware of the use of MCTS ID. Similarly, Nayaket al.(4)reported that majority of the ANMs had no knowledge about ID.

In our study, majority of the ANMs told that they got MCTS ID only from the messages, and most of the ASHAs told that they received recorded calls irregularly. This is in contrary to the findings of Nayaket al.(4)

This study found that MCTS portal was slightly different from $\mathrm{RCH}$ register, which might be a reason for the mistakes found in $\mathrm{RCH}$ register. Nagarajanet al.(5)supports the above finding. The present study also noted that MCTS portal was being converted into $\mathrm{RCH}$ portal, because of which the server was not supporting and working properly with difficulties in uploading the data.

The present study showed that ASHAs had not received any formal training for this program, whereas majority of the ANMs had received training on filling MCTS register. Lack of training regarding filling the register was found to be a major cause of errors in the register. Other studies had also reported the same. $(5,6,7)$ It has been suggested that the performance of MCTS can be improved if ASHAs and ANMs receive proper training to fill the register, and the higher authorities evaluate them from time to time. Our study also found that there was no dedicated DEO in the selected PHCs for entry of data in MCTS portal, which caused delay in data entry. This finding corroborates with other studies. $(5,8)$ Additional data entry staff can help in data entry and reduce workload. 
This study found that internet connectivity and power supply were irregular at all the selected PHCs. This is almost similar to the findings of Nagarajanet al,(5)who reported that poor support in term of internet connectivity and electricity supply were the major challenges in rural areas. It is suggested that adding a component for offline data entry procedure followed by online uploading to the server would be useful.

In this study, DEOs informed that the portal was not connected with other blocks and districts. Most of them said that there was often duplication of data. Similar findings were found by Nayaket al.(4) and Sharma et al.(8) Moreover, this might be a major reason for loss to followup of the women registered under MCTS. It is suggested that if the portal can be connected to other blocks and districts, then the duplication of data can be avoided.

This study showed that ASHAs and ANMs were not receiving any kind of feedback from the higher authority regarding the mistakes made in the register, due to which they were unable to improve their mistakes. Nagarajanet al,(5) also reported that the data were rarely used for decision making. It is suggested that the meeting and training sessions can be used for providing feedback to fill MCTS register or tracking format correctly which in turn will reduce mistakes and also boost confidence in ANMs and ASHAs.

In our study, majority of the ASHAs and ANMs were facing problems in getting the ID. DEOs did not provide MCTS ID immediately. This finding is coherent to the study by Nagarajanet al.(5)

Most of the ASHAs and ANMs were complaining that the duelist was being prepared by them as it was not being provided through the block. Gera et al.(6) reported that MCTS work plans rarely had been used by ANMs.

All DEOs complained about incompleteness of the registers. This result is similar to the finding of Nagarajanet al.(5) It is suggested that training can be arranged for ASHAs to improve their performance.

According to ASHAs and ANMs, they had to go the block PHC several times, which affected their fieldwork and put financial burden on them. This finding is similar to that of Nagarajanet al.,(5)where ANMs reported the same. In this study, it was found that ASHAs and ANMs had to maintain many registers for which they spent daily two to three hours. This finding is coherent to the study by Nayaket al., (4) where majority of the ANMs entered MCTS data regularly. The finding of this study that ASHAs and ANMs had to fill work plans, registers and tracking format separately which caused additional work burden on them, is coherent with that of Gera et al. (6)
This study had found that ASHAs were writing their own mobile numbers in the register to avoid delay in uploading the numbers of pregnant women on the portal. This finding is similar to the study by Sharma et al. (8)

\section{Conclusion}

An effective health system requires a properly functioning health management information system (HMIS). The findings of the current study indicated that inappropriate training of ASHAs, overburdened DEOs and ANMs, poor internet connection at PHC and block, slow server speed, software problem and frequent power failures were major factors affecting the process and performance of MCTS. Lack of timely reporting was revealed as a major limitation. However, MCTS has led to improved accountability and supervision of HCPs. The most effective measure would be to properly train grass root level workers so that more efficient data can be fed into MCTS. Endnote: Due to the changing data requirements of the Reproductive and Child Health ( $\mathrm{RCH}$ ) program, the Ministry has designed $\mathrm{RCH}$ portal.

\section{Authors Contribution}

All authors have contributed equally.

\section{References}

1. Ministry of Health and Family Welfare, Government of India. Maternal and Child Tracking System Operational Manual.Available from: https://www.nrhmmis.nic.in/Home\%20MCH\%20.

2. Ministry of Electronics and Information Technology, Government of India. Mission Mode Projects. Available from: http://deity.gov.in/content/mission-mode-projects.

3. Department of Health and Family Welfare, Government of India. Maternal Health Program, Chapter-4, Annual Report 2013-14:5158.

4. Nayak M.S.D.P, Veni A.K, Madhavi S., Naidu S.A. A Study on Implementation of Mother and Child Tracking System in Chilakaluripeta Cluster of Guntur District, Andhra Pradesh. Indian Journal of Maternal and Child health 2013; 15(4):1-5.

5. Nagarajan P, Tripathy JP, Goel S. Is mother and child tracking system (MCTS) on the right track? An experience from a northern state of India. Indian J Public Health. 2016 Jan-Mar;60(1):34-9. doi: 10.4103/0019-557X.177298. PubMed PMID: 26911215.[PubMed].

6. Gera R, Muthusamy N, Bahulekar A, Sharma A, Singh P, Sekhar A, Singh V. An in-depth assessment of India's Mother and Child Tracking System (MCTS) in Rajasthan and Uttar Pradesh. BMC Health Serv Res. 2015 Aug 11;15:315. doi: 10.1186/s12913-0150920-2. PubMed PMID: 26259836; PubMed Central PMCID: PMC4530478.[PubMed].

7. Dehury RK, Chatterjee SC. Assessment of health management information system for monitoring of maternal health in Jaleswar Block of Balasore District, Odisha, India. Indian J Public Health. 2018 Oct-Dec;62(4):259-264. doi: 10.4103/ijph.IJPH_203_17. PubMed PMID: 30539886.[PubMed].

8. Sharma A., Shinde A., Kar A. Prevalence in the utilization of text message services under the mother and child tracking system of India: a cross sectional study from Pune district, Maharashtra. India. International Journal of Community Medicine and Public Health 2016; 3(8): 2319-2324. 